\title{
Dissolving and biodegradable microneedle technologies for transdermal sustained delivery of drug and vaccine
}

This article was published in the following Dove Press journal:

Drug Design, Development and Therapy

3 September 2013

Number of times this article has been viewed

\author{
Xiaoyun Hong ${ }^{1,2, *}$ \\ Liangming $\mathrm{Wei}^{3, *}$ \\ Fei $\mathrm{Wu}^{2, *}$ \\ Zaozhan $\mathrm{Wu}^{2}$ \\ Lizhu Chen ${ }^{2}$ \\ Zhenguo Liu' \\ Weien Yuan ${ }^{2}$
}

'Department of Neurology, Xinhua Hospital, Shanghai, People's Republic of China; ${ }^{2}$ School of Pharmacy, Shanghai JiaoTong University, Shanghai, People's Republic of China; ${ }^{3}$ Research Institute of Micro/Nano Science and Technology, Shanghai JiaoTong University, Shanghai, People's Republic of China

*These authors contributed equally to this work
Correspondence: Weien Yuan School of Pharmacy, Shanghai JiaoTong University, 800 Dongchuan Road,

Shanghai 200240, People's

Republic of China

Tel +86 2l 34205072

Email yuanweien@I26.com

Zhenguo Liu

Department of Neurology, Xinhua Hospital, Shanghai JiaoTong University

School of Medicine, 1665 Kongjiang

Road, Shanghai 200092, People's

Republic of China

Tel +86 2l 65790000

Email zhenguoliu20I I@yahoo.com.cn
Abstract: Microneedles were first conceptualized for drug delivery many decades ago, overcoming the shortages and preserving the advantages of hypodermic needle and conventional transdermal drug-delivery systems to some extent. Dissolving and biodegradable microneedle technologies have been used for transdermal sustained deliveries of different drugs and vaccines. This review describes microneedle geometry and the representative dissolving and biodegradable microneedle delivery methods via the skin, followed by the fabricating methods. Finally, this review puts forward some perspectives that require further investigation.

Keywords: microneedle, dissolving, biodegradable, sustained release

\section{Introduction}

Hypodermic needles are used for most biotherapeutic and vaccine injections, ${ }^{1}$ providing a low-cost, rapid, and direct way to deliver almost any type of molecule into the body. However, hypodermic needles are utilized primarily in the clinic or at home by patients who have received special training, and safe needle disposal ${ }^{2}$ and other issues such as transportation are problems. ${ }^{3}$ Conventional transdermal drug delivery offers many important advantages, such as accessibility, safety, painless drug administration, potential for self-administration, and avoidance of enzymatic degradation in the gastrointestinal tract or liver, but shows low bioavailability and poor permeability of drugs because of the physical barrier of the stratum corneum. ${ }^{4,5}$ For parenteral controlled-release formulations, in situ-forming implants are also attractive alternatives to preformed implants and microparticles, avoiding the use of large needles or microsurgery and with relatively low cost of manufacture. Such a system stays in a solid state before administration and becomes a hydrogel in situ after injection into the body. However, key issues remain to be solved, including variability of the implant shape and structure, avoidance of burst release during implant formation, and toxicity issues, thus these are far from practical use. ${ }^{6}$ Recently, Peng et al combined nanoparticle and thermosensitive hydrogel technologies in an appropriate manner, which would be a promising system for longer sustained and controlled drug delivery. ${ }^{7}$ However, compared to microneedle technologies, the promising system might have lower patient compliance and transportation convenience. Deadman et al reported more recently that an in situ-forming drug depot, Eligard, was approved by the US Food and Drug Administration. Upon administration, the water-miscible organic solvent dissipates into the surrounding tissue, which leads to polymer precipitation into a depot at the site of injection, entrapping the drug. The obvious disadvantages are the fact that an 
organic solvent is administered (which can lead to toxicity) and the variable shape and size of the implant formed in vivo, which leads to variable rates of drug release. ${ }^{8}$

Microneedle technologies, which were first conceptualized for drug delivery many decades ago, overcome the shortages and preserve the advantages of hypodermic needles and conventional transdermal drug-delivery systems to some extent. They are also superior to in situ-forming implants and in situ-forming drug depot in some fields. Microneedles are needle-like structures with diameters in the size order of microns and lengths up to $900 \mu \mathrm{m} .{ }^{9}$ These structures are used to pierce the upper layer of the skin to enhance transdermal drug delivery by enabling the transport of a host of molecules that cannot be delivered across the skin by passive diffusion alone. ${ }^{10}$ There is no size limit for delivery of macromolecules through these microchannels, as the size of these microchannels is in microns and the maximum dimension of typical macromolecules administered into the body are in nanometers, ${ }^{11}$ thus microneedles can be used to deliver such macromolecules as insulin, growth hormones, immunobiologicals, proteins, and peptides. ${ }^{12}$

Microneedle technologies can be divided into several categories: solid microneedles for skin pretreatment to increase skin permeability, microneedles coated with drugs, polymer microneedles that encapsulate drugs and fully or partially dissolve in the skin, and hollow microneedles for drug infusion into the skin. ${ }^{13,14}$
Microneedles are mostly applied for the transdermal delivery of drugs and vaccines that may require long exposure, among which the dissolving and biodegradable microneedle technologies are most commonly seen. Compared to bolus delivery, the sustained delivery of drug and vaccine is more complicated and is a trend in microneedle technology, thus this article reviews this body of work.

\section{Drug and vaccine delivery methods via the skin \\ Skin microanatomy and the geometry of microneedles}

The stratum corneum is $15-20 \mu \mathrm{m}$ thick and is indispensable as a barrier. The viable epidermis is located below the stratum corneum, and has a thickness of 130-180 $\mu \mathrm{m}$. Below the epidermis lies the dermis, which is about 2,000 $\mu \mathrm{m}$ thick and contains nerves, blood vessels, nociceptors, lymph vessels, hair follicles, and sweat glands (Figure 1). ${ }^{15}$ The epidermal or epithelial layer is a covering carried in the superficial dermis or superficial lamina in the superficial plexus. In the case of skin as a model, the plexus is the intradermal plexus. There are some lymphatic vessels in that layer. In the deep dermal layer or deep lamina, the deep plexus is carried. In the case of skin, this is the subdermal plexus. In that layer, there are most of the lymphatic vessels, and the collagen content of that layer is much greater than in the superficial layer. ${ }^{16}$ It

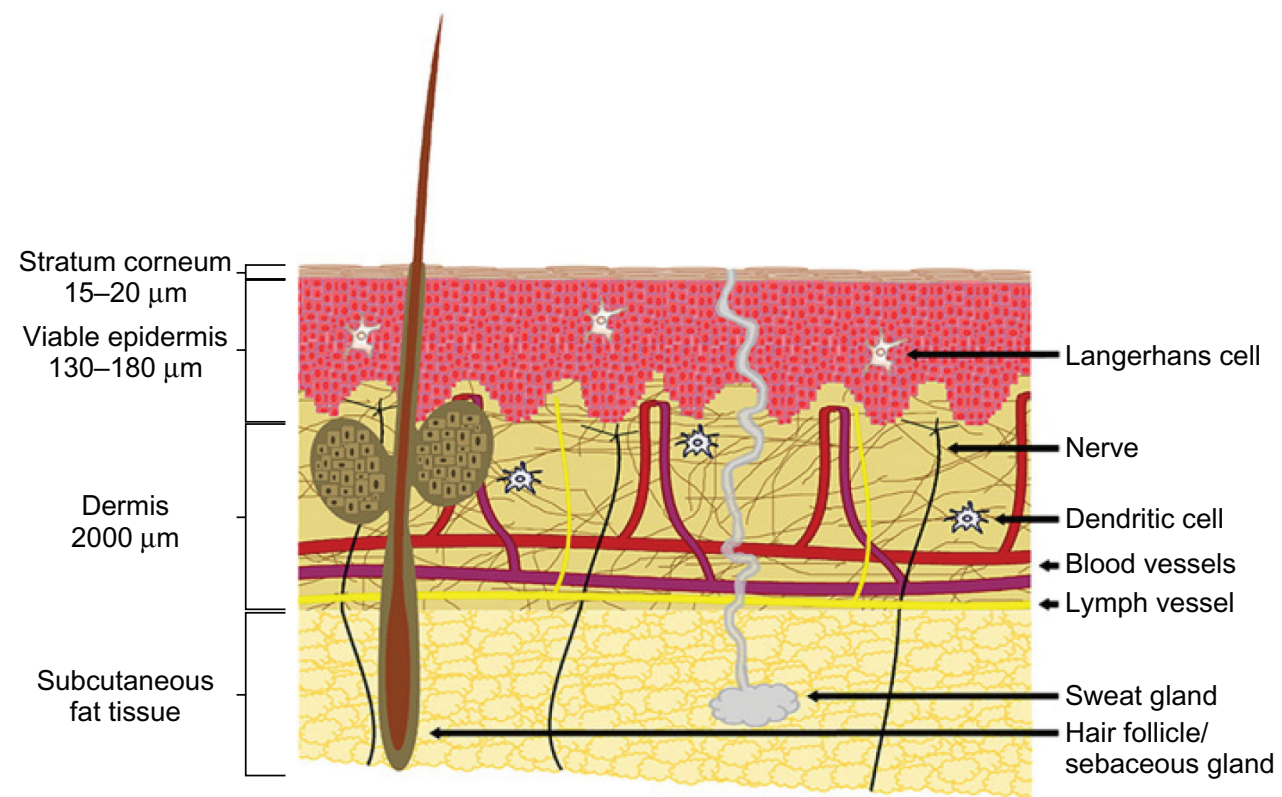

Figure I Skin microanatomy.

Note: () 2012 Elsevier Limited. Reproduced with permission from van der Maaden K, Jiskoot W, Bouwstra J. Microneedle technologies for (trans)dermal drug and vaccine delivery. J Control Release. 20 I2; I6I (2):645-655. ${ }^{15}$ 
has long been recognized as a highly immune reactive tissue containing an abundance of antigen-presenting cells and immunocompetent cells, especially within the epidermal and dermal skin layers. ${ }^{17,18}$

Skin microanatomy largely determines the geometry of microneedles. Yana et al proved that microneedle arrays with longer needles $(>600 \mu \mathrm{m})$ were more effective in creating pathways across skin and enhancing drug flux, and microneedle arrays with lower needle densities $\left(<2,000\right.$ needles $\left./ \mathrm{cm}^{2}\right)$ were more effective in enhancing drug flux if the microneedles had sufficient length $(>600 \mu \mathrm{m}) .{ }^{19}$ Noh et $\mathrm{al}^{20}$ measured skin irritation using microneedles of $500 \mu \mathrm{m}$ depth, and concluded that there was little difference in the decrease of redness after microneedle application based on application time, but redness was generally maintained until 30 minutes and rapidly decreased between 30 minutes and 2 hours. Gomaa et $\mathrm{al}^{21}$ found that when $600 \mu \mathrm{m}$-long microneedles were applied to the dermal side of skin, the transepidermal water-loss measurements remained at baseline levels. This is explainable by the fact that typically only $10 \%-30 \%$ of the needle length actually penetrates tissue. ${ }^{22}$ Gomaa et $\mathrm{al}^{21}$ also found that increasing microneedle density introduced complex effects. Firstly, more skin channels may have been created, and these inhibited the long-term, tissue contraction-mediated partial recovery of the barrier. However, there was also some evidence that at high microneedle densities, individual needles may no longer effectively breach the barrier due to a "bed of nails" effect. Leaving the array embedded in the skin seemed to diminish the magnitude of the initial postinsertion drop in barrier function. Furthermore, Lee et $\mathrm{al}^{23}$ concluded that pyramidal microneedles were stronger than conical ones, probably due to their larger cross-sectional area at the same base width/diameter.

\section{Dissolving microneedles}

Dissolving microneedles, mostly using different kinds of sugars as the matrix (Figure 2), usually release drugs or vaccines quickly in vivo, ${ }^{24} \mathrm{eg}$, Ito et $\mathrm{al}^{25}$ reported that insulin released from microneedles very quickly, and almost all of the formulated insulin was released within 1 hour when dextrin was used as matrix. Nevertheless, sustained release of drugs or vaccines is also required under some circumstances. Lee et $\mathrm{al}^{23}$ prepared microneedles with model drug encapsulated not within the microneedle tips but only in the backing layer, which served as a controlled-release reservoir that delivered molecules by a combination of swelling the backing with interstitial fluid drawn out of the skin and molecule diffusion into the skin via channels formed by dissolved microneedles. They revealed that sulforhodamine release from carboxymethyl cellulose microneedle patches exhibited an initial lag time of a few hours, followed by steady release for approximately 1 day. Similar behavior was seen for microneedle patches made of amylopectin, but with slower kinetics. In this case, lag time was longer and release took place over a few days. Polymeric dissolving microneedles designed by Donnelly et al have been produced from Gantrez AN-139, and delivered $83 \%$ of the encapsulated theophylline into porcine skin within 24 hours. $^{26}$

Kumar et $\mathrm{a}^{27}$ characterized and used maltose microneedles to microporate full-thickness pig-ear skin to evaluate drug delivery of model small (calcein) and large (human growth hormone) molecules. It was found that modulated
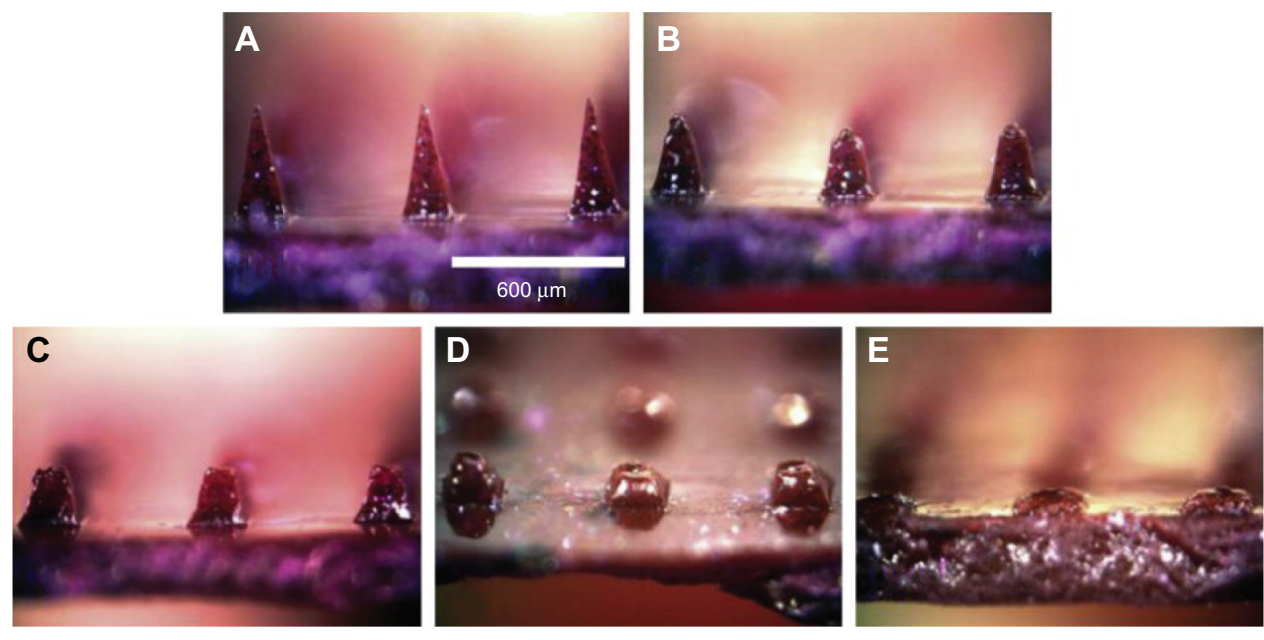

Figure 2 (A-E) Commonly seen dissolving microneedles and the drug-release procedure.

Note: (C) 2008 Elsevier Limited. Reproduced with permission from Lee JW, Park JH, Prausnitz MR. Dissolving microneedles for transdermal drug delivery. Biomaterials. 2008;29(I3):21 I3-21 $24 .^{23}$ 
transdermal delivery of small as well as large molecules is possible upon microporation of the skin in combination with iontophoresis (ITP). The modulated ITP protocol resulted in peaks in flux with application of current and gradual decrease with termination of current, and current density and time could be used appropriately to program a desired drug-delivery profile. Garland et $\mathrm{al}^{28}$ studied the potential for ITP to be combined with polymeric microneedle devices that remain in contact with the skin during the course of drug delivery procedure for the first time. Furthermore, it has been shown that the application of an electric current enables the permeation of macromolecules from the entire microneedle-array matrix, and not just that which was contained within the microneedles alone. Thus the application of an electric current significantly increased the extent of macromolecular delivery from the poly(methyl vinyl ether maleic anhydride) microneedle array, which is also promising for the sustained delivery of drugs and vaccines. Wu et $\mathrm{al}^{29}$ reported a similar finding, wherein the transdermal permeation of high-molecular-weight compounds through microneedle-induced channels could be increased through the combination of ITP. Ito et al reported sustained-release self-dissolving micropiles (SDMPs), in which porous silicon dioxide and porous calcium silicate were used as nanoporous microparticles to adsorb insulin, and the microparticleadsorbed insulin was molded to SDMPs using chondroitin sulfate as a base. They concluded that long-acting SDMP preparation would be possible by means of porous silicate adsorbent-held insulin. ${ }^{30}$

\section{Biodegradable microneedles}

Biodegradable microneedles, mostly using different kinds of biodegradable polymers, including polylactic acid, chitosan, polyglycolic acid, or poly(lactide-co-glycolide) (PLGA) to form the matrix, degrade in the skin after application, whereby the release of incorporated drugs can be sustained for months by choosing the proper polymer. A previous study demonstrated the possibility of these biodegradable microneedles as a patient-friendly substitute for conventional sustained-delivery methods. ${ }^{31}$ However, these microneedles must be inserted and remain in the skin for several days to utilize the degradation property of biodegradable polymer effectively.

Kim et $\mathrm{al}^{32}$ demonstrated that microneedle separation into the skin was mediated by hydrogel swelling in response to contact with body fluid after the needles were inserted into the skin. The hydrogel particles absorbed water quickly, resulting in the cracking of the microneedles due to the difference

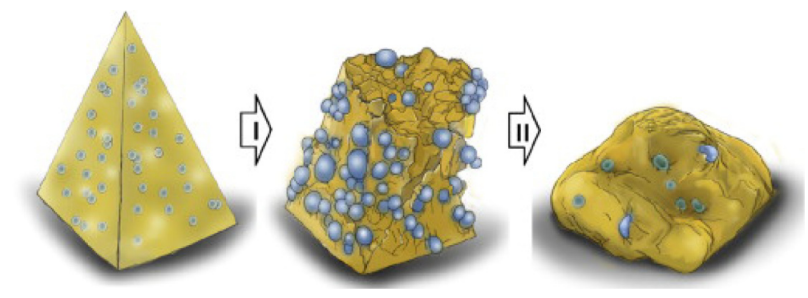

Figure 3 Microneedle separation into the skin was mediated by hydrogel swelling. Note: (C) 2012 Elsevier Limited. Reproduced with permission from Kim M, Jung B, Park JH. Hydrogel swelling as a trigger to release biodegradable polymer microneedles in skin. Biomaterials. 20I2;33(2):668-678. ${ }^{32}$

in volume expansion between the needle-matrix polymer and the hydrogel particles. The swollen particles caused the microneedles to totally break down, leaving the microneedle tips in the porcine cadaver skin in vitro and in the hairless mouse skin in vivo (Figure 3 ). Chu et al ${ }^{33}$ introduced separable arrowhead microneedles that featured micron-size sharp tips mounted on blunt shafts. Upon insertion in the skin, the sharp-tipped polymer arrowheads encapsulating drug separate from their metal shafts and remain embedded in the skin for subsequent dissolution and drug release. The blunt metal shafts can then be discarded. Due to rapid separation of the arrowhead tips from the shafts within seconds, administration using arrowhead microneedles can be carried out rapidly, while drug-release kinetics can be independently controlled, based on separable arrowhead formulation (Figure 4). Park et $\mathrm{al}^{31}$ developed arrays of microneedles that were fabricated out of PLGA using a mold-based technique to encapsulate model drugs - calcein and bovine serum albumin - either as a single encapsulation within the needle matrix or as a double encapsulation, by first encapsulating the drug within carboxymethyl cellulose or poly-L-lactide microparticles and then encapsulating drug-loaded microparticles within needles. In vitro release of calcein and bovine serum albumin from three different encapsulation formulations was measured over time, and was shown to be controlled by the encapsulation method to achieve release kinetics ranging from hours to months. Tsioris et $\mathrm{al}^{34}$ reported on a fabrication method to produce silk biopolymer microstructures with
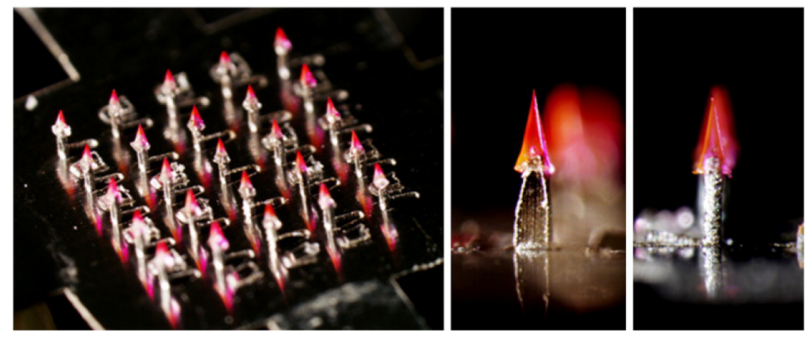

Figure 4 Separable arrowhead microneedles.

Note: (C) 20I I Elsevier Limited. Reproduced with permission from Chu LY, Prausnitz MR. Separable arrowhead microneedles. J Control Release. 201 I;149(3):242-249.33 
the high aspect ratios required to manufacture microneedle systems. Room temperature and aqueous-based micromolding allow for the bulk loading of these microneedle structures with temperature-sensitive drugs such as peptides, antibiotics, and vaccines, or any temperature-labile therapeutic. Controlled release of a model drug is achieved by adjusting the postprocessing conditions of the microneedle structures, mainly by controlling the silk protein secondary structure.

Ausiello et $\mathrm{al}^{35}$ released a method for extended and controlled delivery of parathyroid hormone to a patient in need, involving implanting a medical device into the patient, the medical device comprising a substrate, a plurality of reservoirs in the substrate, and a release system contained in each of the reservoirs, wherein the release system comprised parathyroid hormone, and controllably releasing a pharmaceutically effective amount of the parathyroid hormone from the reservoirs.

Microneedle delivery of nucleic acids, in particular plasmid DNA (pDNA), to the skin represents a potential new approach for the clinical management of genetic skin diseases and cutaneous cancers, and for intracutaneous genetic immunization. DNA vaccines have many potential benefits, but have failed to generate robust immune responses in humans. ${ }^{36}$ DeMuth et $\mathrm{al}^{37}$ reported an approach for rapid implantation of vaccine-loaded polymer films carrying DNA, immune-stimulatory RNA, and biodegradable polycations using microneedles coated with releasable polyelectrolyte multilayers that promoted local transfection and controlled the persistence of DNA and adjuvants in the skin from days to weeks, with kinetics determined by the film composition. These "multilayer tattoo" DNA vaccines induced immune responses against a model HIV antigen comparable to electroporation in mice, enhanced memory T-cell generation, and elicited 140-fold higher gene expression in nonhuman primate skin than intradermal DNA injection, indicating the potential of this strategy for enhancing DNA vaccination
(Figure 5). Marc Pearton et $\mathrm{al}^{38}$ proved that the pDNA-coated microneedles facilitated reporter-gene expression in viable human skin, whilst the efficiency of gene expression from coated microneedles will depend upon suitable DNA loading, efficient and reproducible skin puncture, and rapid in situ dissolution of the plasmid at the site of delivery.

\section{Microneedle-fabricating methods}

Fabrication of microneedles usually follows the steps of molding, preparation of microneedle matrix, casting, removal, and drying. ${ }^{39}$ The concrete methods are determined by the desired property of the microneedles. However, harsh fabrication methods (ie, using high temperature or organic solvent) may damage temperature-sensitive drugs, particularly peptides and proteins, so this review mainly focuses on mild fabrication methods. Vacuum and centrifugation are often applied in casting the microneedle matrix into the molds. Whilst a polydimethylsiloxane micromold can be created with precise morphological fidelity to master microneedle structures, sugar solutions did not completely fill the micromold invaginations, due to the high surface tension of the solution. Martin et $\mathrm{al}^{40}$ revealed a simple and novel low-temperature vacuum-deposition micromolding methodology for biodegradable sugar-glass microneedle fabrication. In the original vacuum-oven method, vacuum pressure could not be applied before the sugar solution was placed on the mold surface. Subsequently, when the droplet of sugar solution was applied, air was entrapped within the micromold invaginations. An optimized method was developed whereby a vacuum was produced within an enclosed chamber before the sugar solution was applied to the mold surface.

DeMuth et $\mathrm{al}^{41}$ developed microneedle-fabrication methods featuring drug-loaded PLGA microparticles or solid PLGA tips. PLGA microparticles were applied to the surface of the mold in an aqueous suspension and compacted into the mold cavities through centrifugation. Excess
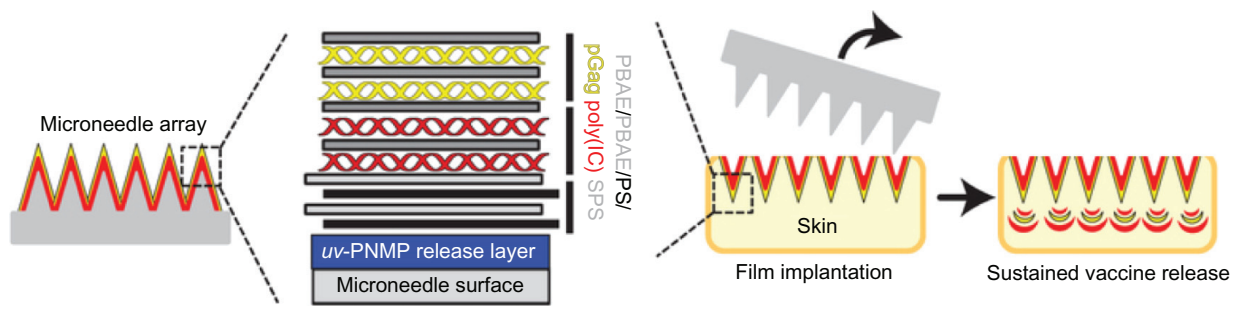

Figure 5 Sustained vaccine release from "multilayer tattoo" microneedles.

Note: (C) 2013 Elsevier Limited. Reproduced with permission from DeMuth PC, Min Y, Huang B, et al. Polymer multilayer tattooing for enhanced DNA vaccination. Nat Mater. 2013;12(4):367-376. ${ }^{37}$

Abbreviations: uv-PNMP, poly(o-nitrobenzyl-methacrylate-co-methyl-methacrylate-co-poly(ethylene-glycol)-methacrylate) on brief exposure to ultraviolet; PBAE, biodegradable poly( $\beta$-amino-ester); PS, protamine-sulphate; pGag, gag polyprotein; poly(l:C), polyriboinosinic polyribocytidylic acid; SPS, poly(4-styrene-sulphonate). 
microparticles were then removed, and the microparticleloaded mold was allowed to dry. Then, a concentrated aqueous solution of poly(acrylic acid) was added to the mold surface and infiltrated into the packed PLGA particle bed via centrifugation. After being dried under vacuum, a solid PLGA-poly(acrylic acid) microparticle matrix was obtained. If the microparticle-loaded polydimethylsiloxane molds were dried and incubated under vacuum to fuse the embedded PLGA particles, solidified PLGA tips were obtained. DeMuth et $\mathrm{al}^{37}$ demonstrated a new approach for DNA vaccination via multilayer "tattooing," using microneedles employing a pH-responsive release layer to implant biodegradable vaccine-loaded polymer films rapidly into the skin.

Mefti et al released a patent concerning the administration of drugs with microneedles. More specifically, it concerned microneedles comprising a part that dissolves by hydrolysis once they have penetrated the skin. ${ }^{42}$

It can also be concluded that enhancing methods such as ITP, as well as with different drug carriers (eg, microand nanoparticles) will be applied to the fabrication of microneedles. ${ }^{43}$

\section{Perspectives}

Microneedles effectively circumvent the skin barrier to offer this route as a potential alternative to oral and parenteral delivery of therapeutics, ${ }^{44}$ and relatively large doses can be administered due to bulk loading of dissolvable or biodegradable systems. However, there are still several problems that remain to be solved. For example, the barrier function of the skin changes from one site to another on the same person, from person to person, and with age. ${ }^{45}$ It is important to ensure that patients obtain the same and required dose during each microneedle administration, since the difference in individual skin and the penetration depth of microneedles relate to the stress upon the skin, thus an applicator may be required in order to obtain a reproducible penetration depth. Besides, the sustained release of drugs or vaccines is more complicated than bolus release, and the kinetics should be further investigated and ensured. It is also necessary to investigate whether dissolved or degraded matrices have side effects if microneedles are used frequently. Although these materials are commonly seen excipients in drugs, the accumulation levels and their influences should be noted for prudential reasons. In addition, in some microneedle-fabrication cases, the polymer-melting temperatures were above $135^{\circ} \mathrm{C}$ and vacuum necessary for processing, and these conditions could be detrimental to various temperature-sensitive drugs, particularly peptides and proteins. Polymer microneedles for controlled-release delivery are also constrained by needle mechanical properties when the main matrix is PLGA or carboxymethyl cellulose. Park et al found that microneedles with $2 \%$ drug loading retained sufficient mechanical strength, but needles with $10 \%$ loading did not. Although the maximum dose constrains applications, controlled-release delivery of up to $1 \mathrm{mg}$ has a number of candidate drugs on the market, with more likely to be approved in the future. ${ }^{31}$

DNA vaccines have been intensively studied because of potential advantages, such as ease of good-manufacturingpractice production, lack of antivector immunity, and the capability to promote both cellular and humoral immune responses. ${ }^{46,47}$ Since the discovery ${ }^{48}$ that genetically engineered DNA can be delivered in vaccine form and elicit an immune response, there has been much progress in understanding the basic biology of this platform. ${ }^{49,50}$ A large amount of data has been generated in preclinical model systems, ${ }^{51,52}$ and more sustained cellular responses and more consistent antibody responses are being observed in the clinic. ${ }^{53,54}$ Microneedle application is promising in promoting local transfection and controlling the persistence of DNA and adjuvants in the skin from days to weeks to function as an optimal strategy for safe, reproducible, and pain-free DNA vaccination. For therapeutic proteins, preservation of protein structure during manufacture, storage, and use is considered even more important than for vaccines, and unwanted immunogenicity can lead to total loss of the therapeutic effect of a protein by neutralizing antibodies, and may even lead to depletion of endogenous proteins or breaking the immune tolerance to self-antigens. For dissolving microneedles, future studies will partially focus upon the incorporation of other bioactivity-enhancing methods including ITP components into polymeric microneedle devices and investigation of their potential for efficient, electrically controlled pulsatile delivery of macromolecules from drug-loaded dissolving polymeric microneedle arrays. ${ }^{29,35}$

Altogether, dissolving and biodegradable microneedle technologies have a bright future for transdermal sustained delivery of drug and vaccine, and require further studies. ${ }^{55}$

\section{Acknowledgments}

The study was supported by the Projects of National Science Foundation of China (81373366, 81071025 and 81171203), and Projects of the Shanghai Committee of Science and Technology, People's Republic of China (11nm0503300, 11410708900, and 12XD1403800). The authors thank the Analytical Center of Shanghai JiaoTong University for technical support. 


\section{Disclosure}

The authors report no conflicts of interest in this work.

\section{References}

1. Arendt-Nielsen L, Egekvist H, Bjerring P. Pain following controlled cutaneous insertion of needles with different diameters. Somatosens Mot Res. 2006;23(1-2):37-43.

2. Tarnow K, King N. Intradermal injections: traditional bevel up versus bevel down. Appl Nurs Res. 2004;17(4):275-282.

3. Giudice EL, Campbell JD. Needle-free vaccine delivery. Adv Drug Deliver Rev. 2006;58(1):68-89.

4. Arora A, Prausnitz MR, Mitragotri S. Micro-scale devices for transdermal drug delivery. Int J Pharm. 2008;364(2):227-236.

5. Prausnitz MR, Langer R. Transdermal drug delivery. Nat Biotechnol. 2008;26(11):1261-1268

6. Kempe S, Mäder K. In situ forming implants - an attractive formulation principle for parenteral depot formulations. J Control Release. 2012; 161(2):668-679.

7. Peng $Q$, Sun $X$, Gong T, et al. Injectable and biodegradable thermosensitive hydrogels loaded with PHBHHx nanoparticles for the sustained and controlled release of insulin. Acta Biomater. 2013;9(2):5063-5069.

8. Deadman CM, Kellaway IW, Yasin M, Dickinson PA, Murdan S. An investigation into the influence of drug lipophilicity on the in vivo absorption profiles from subcutaneous microspheres and in situ forming depots. J Control Release. 2007;122(1):79-85.

9. Donnelly RF, Garland MJ, Morrow DIJ, et al. Optical coherence tomography is a valuable tool in the study of the effects of microneedle geometry on skin penetration characteristics and in-skin dissolution. J Control Release. 2010;147(3):333-341.

10. Ghosh P, Pinninti RR, Hammell DC, Paudel KS, Stinchcomb AL. Development of a codrug approach for sustained drug delivery across microneedle-treated skin. J Pharm Sci. 2013;102(5):1458-1467.

11. Felton LA. Transdermal and intradermal delivery of therapeutic agents application of physical technologies. Drug Dev Ind Pharm. 2012; 38(4):513.

12. Bariya SH, Gohel MC, Mehta TA, Sharma OP. Microneedles: an emerging transdermal drug delivery system. J Pharm Pharmacol. 2012; 64(1):11-29.

13. Gupta J, Park SS, Bondy B, Felner EI, Prausnitz MR. Infusion pressure and pain during microneedle injection into skin of human subjects. Biomaterials. 2011;32(28):6823-6831.

14. Hirobe $\mathrm{S}$, Okada N, Nakagawa S. Transcutaneous vaccines - current and emerging strategies. Expert Opin Drug Deliv. 2013;10(4):485-498.

15. van der Maaden K, Jiskoot W, Bouwstra J. Microneedle technologies for (trans)dermal drug and vaccine delivery. $J$ Control Release. 2012;161(2):645-655.

16. Jordan GH. Techniques of tissue handling and transfer. J Urol. 1999; 162(3):1213-1217.

17. Lambert PH, Laurent PE. Intradermal vaccine delivery: will new delivery systems transform vaccine administration? Vaccine. 2008;26(26): 3197-3208.

18. Nicolas JF, Guy B. Intradermal, epidermal and transcutaneous vaccination: from immunology to clinical practice. Expert Rev Vaccines. 2008;7(8):1201-1214.

19. Yan G, Warner KS, Zhang J, Sharma S, Gale BK. Evaluation needle length and density of microneedle arrays in the pretreatment of skin for transdermal drug delivery. Int J Pharm. 2010;391(1-2):7-12.

20. Noh YW, Kim TH, Baek JS, et al. In vitro characterization of the invasiveness of polymer microneedle against skin. Int $J$ Pharm. 2010;397(1-2):201-205.

21. Gomaa YA, Morrow DI, Garland MJ, Donnelly RF, El-Khordagui LK, Meidan VM. Effects of microneedle length, density, insertion time and multiple applications on human skin barrier function: assessments by transepidermal water loss. Toxicol In Vitro. 2010;24(7): 1971-1978.
22. Martanto W, Moore JS, Couse T, Prausnitz MR. Mechanism of fluid infusion during microneedle insertion and retraction. J Control Release. 2006;112(3):357-361.

23. Lee JW, Park JH, Prausnitz MR. Dissolving microneedles for transdermal drug delivery. Biomaterials. 2008;29(13):2113-2124.

24. Migalska K, Morrow DI, Garland MJ, Thakur R, Woolfson AD, Donnelly RF. Laser-engineered dissolving microneedle arrays for transdermal macromolecular drug delivery. Pharm Res. 2011;28(8): 1919-1930.

25. Ito Y, Hagiwara E, Saeki A, Sugioka N, Takada K. Feasibility of microneedles for percutaneous absorption of insulin. Eur J Pharm Sci. 2006;29(1):82-88.

26. Donnelly RF, Majithiya R, Singh TR, et al. Design, optimization and characterisation of polymeric microneedle arrays prepared by a novel laser-based micromoulding technique. Pharm Res. 2011;28(1): $41-57$.

27. Kumar V, Banga AK. Modulated iontophoretic delivery of small and large molecules through microchannels. Int J Pharm. 2012;434(1-2): $106-114$.

28. Garland MJ, Caffarel-Salvador E, Migalska K, Woolfson AD, Donnelly RF. Dissolving polymeric microneedle arrays for electrically assisted transdermal drug delivery. J Control Release. 2012;159(1):52-59.

29. Wu XM, Todo H, Sugibayashi K. Enhancement of skin permeation of high molecular compounds by a combination of microneedle pretreatment and iontophoresis. J Control Release. 2007;118(2):189-195.

30. Ito Y, Hagiwara E, Saeki A, Sugioka N, Takada K. Sustained-release self-dissolving micropiles for percutaneous absorption of insulin in mice. J Drug Target. 2007;15(5):323-326.

31. Park JH, Allen MG, Prausnitz MR. Polymer microneedles for controlled-release drug delivery. Pharm Res. 2006;23(5):1008-1019.

32. Kim M, Jung B, Park JH. Hydrogel swelling as a trigger to release biodegradable polymer microneedles in skin. Biomaterials. 2012;33(2):668-678.

33. Chu LY, Prausnitz MR. Separable arrowhead microneedles. J Control Release. 2011;149(3):242-249.

34. Tsioris K, Raja WK, Pritchard EM, Panilaitis B, Kaplan DL, Omenetto FG. Fabrication of silk microneedles for controlled-release drug delivery. Adv Funct Mater. 2012;22(2):330-335.

35. Ausiello D, Herman SJ, Prescott JH, Santini J, inventors. Method and device for the controlled delivery of parathyroid hormone. United States patent US 20040082937. April 29, 2004.

36. Gill HS, Söderholm J, Prausnitz MR, Sällberg M. Cutaneous vaccination using microneedles coated with hepatitis C DNA vaccine. Gene Ther. 2010;17(6):811-814.

37. DeMuth PC, Min Y, Huang B, et al. Polymer multilayer tattooing for enhanced DNA vaccination. Nat Mater. 2013;12(4):367-376.

38. Pearton M, Saller V, Coulman SA, et al. Microneedle delivery of plasmid DNA to living human skin: formulation coating, skin insertion and gene expression. J Control Release. 2012;160(3):561-569.

39. Olatunji O, Das DB. Drug delivery using microneedles In: MooYoung M, editor. Comprehensive Biotechnology. 2nd ed. Burlington: Academic Press; 2011:625-642.

40. Martin CJ, Allender CJ, Brain KR, Morrissey A, Birchall JC. Low temperature fabrication of biodegradable sugar glass microneedles for transdermal drug delivery applications. $J$ Control Release. 2012;158(1):93-101.

41. Demuth PC, Garcia-Beltran WF, Ai-Ling ML, Hammond PT, Irvine DJ. Composite dissolving microneedles for coordinated control of antigen and adjuvant delivery kinetics in transcutaneous vaccination. Adv Funct Mater. 2013;23(2):161-172.

42. Mefti S, Cachemaille A, Piveteau LD, Lemaire P, inventors. Soluble microneedle. World Intellectual Property Organization patent WO/2011/076537. June 30, 2011

43. Nava-Arzaluz MG, Calderon-Lojero I, Quintanar-Guerrero D, Villalobos-Garcia R, Ganem-Quintanar A. Microneedles as transdermal delivery systems: combination with other enhancing strategies. Current Drug Deliver. 2012;9(1):57-73. 
44. Matteucci M, Fanetti M, Casella M. Poly vinyl alcohol re-usable masters for microneedle replication. Microelectron Eng. 2009;86(4-6):752-756.

45. Sharma A, Saini S, Rana A. Transdermal drug delivery system: a review. Res J Pharm Technol. 2012;5(6):757-763.

46. Chen $\mathrm{CH}$, Wang TL, Hung CF, et al. Enhancement of DNA vaccine potency by linkage of antigen gene to an HSP70 gene. Cancer Res. 2000;60(4):1035-1042.

47. Yang ZY, Kong WP, Huang Y, et al. A DNA vaccine induces SARS coronavirus neutralization and protective immunity in mice. Nature. 2004;428(6982):561-564.

48. Tang DC, DeVit M, Johnston SA. Genetic immunization is a simple method for eliciting an immune response. Nature. 1992;356(6365): $152-154$.

49. Ulmer JB, Wahren B, Liu MA. Gene-based vaccines: recent technical and clinical advances. Trends Mol Med. 2006;12(5):216-222.

50. Peters BS, Jaoko W, Vardas E, et al. Studies of a prophylactic HIV-1 vaccine candidate based on modified vaccinia virus Ankara (MVA) with and without DNA priming: effects of dosage and route on safety and immunogenicity. Vaccine. 2007;25(11):2120-2127.
51. Buchan S, Grønevik E, Mathiesen I, King CA, Stevenson FK, Rice J. Electroporation as a "prime/boost" strategy for naked DNA vaccination against a tumor antigen. J Immunol. 2005;174(10):6292-6298.

52. Jimenez GS, Planchon R, Wei Q, et al. Vaxfectin-formulated influenza DNA vaccines encoding NP and M2 viral proteins protect mice against lethal viral challenge. Hum Vaccin. 2007;3(5):157-164.

53. Kutzler MA, Weiner DB. DNA vaccines: ready for prime time? Nat Rev Genet. 2008;9(10):776-788.

54. Chen D, Maa YF, Haynes JR. Needle-free epidermal powder immunization. Expert Rev Vaccines. 2002;1(3):265-276.

55. Chen $\mathrm{H}, \mathrm{Zhu} \mathrm{H}$, Zheng J, et al. Iontophoresis-driven penetration of nanovesicles through microneedle-induced skin microchannels for enhancing transdermal delivery of insulin. $J$ Control Release. 2009;139(1):63-72.

\section{Publish your work in this journal}

Drug Design, Development and Therapy is an international, peerreviewed open-access journal that spans the spectrum of drug design and development through to clinical applications. Clinical outcomes, patient safety, and programs for the development and effective, safe, and sustained use of medicines are a feature of the journal, which has also been accepted for indexing on PubMed Central. The manuscript management system is completely online and includes a very quick and fair peer-review system, which is all easy to use. Visit http://www.dovepress.com/testimonials.php to read real quotes from published authors.

Submit your manuscript here: http://www.dovepress.com/drug-design-development-and-therapy-journal 